Yi Jin, Tao Fu, Bo-Yang Ma, Ping Wei, Ji-Xing Zhao and Li Zhao*

\title{
Synthesis and crystal structure of 1-\{4-[(3-bromo- 2-hydroxy-benzylidene)amino]phenyl\}ethanone, $\mathrm{C}_{15} \mathrm{H}_{12} \mathrm{BrNO}_{2}$
}

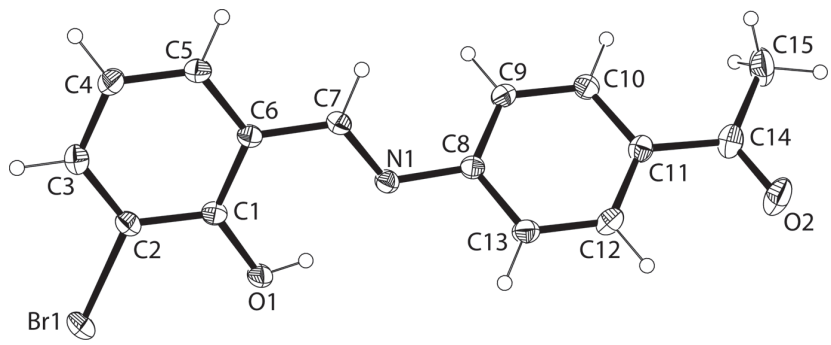

https://doi.org/10.1515/ncrs-2020-0181

Received April 11, 2020; accepted May 11, 2020; available online May 22, 2020
Table 1: Data collection and handling.

\begin{tabular}{ll}
\hline Crystal: & Orange block \\
Size: & $0.19 \times 0.17 \times 0.14 \mathrm{~mm}$ \\
Wavelength: & Mo $K \alpha$ radiation $(0.71073 \AA)$ \\
$\mu:$ & $3.02 \mathrm{~mm}^{-1}$ \\
Diffractometer, scan mode: & Bruker D8 Venture, $\varphi$ and $\omega$ \\
$\theta_{\text {max }}$, completeness: & $26.7^{\circ},>99 \%$ \\
$N(h k l)_{\text {measured }}, N(h k l)_{\text {unique }}, R_{\text {int }}:$ & $16513,2878,0.053$ \\
Criterion for $I_{\text {obs }}, N(h k l)_{\mathrm{gt}}:$ & $I_{\text {obs }}>2 \sigma\left(I_{\text {obs }}\right), 2268$ \\
$N(\text { param })_{\text {refined }}:$ & 177 \\
Programs: & Bruker [1], SHELX [2], Olex2 [3] \\
\hline
\end{tabular}

Table 2: Fractional atomic coordinates and isotropic or equivalent isotropic displacement parameters $\left(\AA^{2}\right)$.

\begin{tabular}{lrrrr}
\hline Atom & $\boldsymbol{x}$ & $\boldsymbol{y}$ & $\boldsymbol{z}$ & $\boldsymbol{U}_{\text {iso }}{ }^{*} \boldsymbol{U}_{\text {eq }}$ \\
\hline Br1 & $0.67409(2)$ & $0.99765(3)$ & $0.31965(2)$ & $0.04061(10)$ \\
O1 & $0.49403(15)$ & $0.79837(16)$ & $0.34311(14)$ & $0.0313(4)$ \\
H1 & $0.442(2)$ & $0.752(2)$ & $0.356(3)$ & $0.048(8)^{\star}$ \\
O2 & $-0.10570(16)$ & $0.32640(19)$ & $0.3465(2)$ & $0.0528(5)$ \\
N1 & $0.37801(17)$ & $0.66521(18)$ & $0.45082(17)$ & $0.0271(4)$ \\
C1 & $0.56172(19)$ & $0.8472(2)$ & $0.4488(2)$ & $0.0261(5)$ \\
C2 & $0.6513(2)$ & $0.9415(2)$ & $0.4576(2)$ & $0.0285(5)$ \\
C3 & $0.7228(2)$ & $0.9937(2)$ & $0.5644(2)$ & $0.0331(5)$ \\
H3 & 0.782949 & 1.058198 & 0.568244 & $0.040^{*}$ \\
C4 & $0.7074(2)$ & $0.9527(3)$ & $0.6659(2)$ & $0.0343(5)$ \\
H4 & 0.756546 & 0.988796 & 0.739310 & $0.041^{*}$ \\
C5 & $0.6200(2)$ & $0.8590(2)$ & $0.6594(2)$ & $0.0311(5)$ \\
H5 & 0.609738 & 0.830376 & 0.729038 & $0.037^{*}$ \\
C6 & $0.5461(2)$ & $0.8053(2)$ & $0.5520(2)$ & $0.0256(5)$ \\
C7 & $0.45130(19)$ & $0.7117(2)$ & $0.5481(2)$ & $0.0265(5)$ \\
H7 & 0.443319 & 0.684266 & 0.619058 & $0.032^{*}$ \\
C8 & $0.2853(2)$ & $0.5751(2)$ & $0.4493(2)$ & $0.0258(5)$ \\
C9 & $0.3046(2)$ & $0.4722(2)$ & $0.5298(2)$ & $0.0295(5)$ \\
H9 & 0.382069 & 0.461217 & 0.589869 & $0.035^{*}$ \\
C10 & $0.2106(2)$ & $0.3859(2)$ & $0.5221(2)$ & $0.0305(5)$ \\
H10 & 0.223952 & 0.316030 & 0.577273 & $0.037^{*}$ \\
C11 & $0.0963(2)$ & $0.4005(2)$ & $0.4341(2)$ & $0.0297(5)$ \\
C12 & $0.0792(2)$ & $0.5030(2)$ & $0.3535(2)$ & $0.0349(5)$ \\
H12 & 0.001914 & 0.513855 & 0.293061 & $0.042^{*}$ \\
C13 & $0.1724(2)$ & $0.5889(2)$ & $0.3600(2)$ & $0.0320(5)$ \\
H13 & 0.159641 & 0.657280 & 0.303574 & $0.038^{*}$ \\
C14 & $-0.0070(2)$ & $0.3094(2)$ & $0.4230(3)$ & $0.0371(6)$ \\
& & & &
\end{tabular}

*Corresponding author: Li Zhao, School of Chemical and Biological Engineering, Lanzhou Jiaotong University, Lanzhou 730070, P.R. China, e-mail: zhaoli_72@163.com. https://orcid.org/00000002-9898-9552

Yi Jin, Tao Fu, Bo-Yang Ma and Ping Wei: School of Chemical and Biological Engineering, Lanzhou Jiaotong University, Lanzhou 730070, P.R. China

Ji-Xing Zhao: Shihezi University, Analysis and Testing Center, Xinjiang 832003, P.R. China 
Table 2 (continued)

\begin{tabular}{lrrrr}
\hline Atom & $\boldsymbol{x}$ & $\boldsymbol{y}$ & $\boldsymbol{z}$ & $\boldsymbol{U}_{\text {iso }}{ }^{*} / \boldsymbol{U}_{\text {eq }}$ \\
\hline C15 & $0.0122(2)$ & $0.1955(3)$ & $0.5062(3)$ & $0.0544(8)$ \\
H15A & -0.063379 & 0.144533 & 0.486341 & $0.082^{*}$ \\
H15B & 0.035579 & 0.229468 & 0.586179 & $0.082^{*}$ \\
H15C & 0.076944 & 0.138170 & 0.500507 & $0.082^{*}$ \\
\hline
\end{tabular}

for $\mathrm{C}_{15} \mathrm{H}_{12} \mathrm{BrNO}_{2}$ : C, $56.62 \% ; \mathrm{H}, 3.80 \%$;, $4.40 \%$. Found: C, $56.85 \%$; H, 3.97\%; N, 4.12\%.

\section{Experimental details}

Hydrogen atoms were placed in their geometrically idealized positions and constrained to ride on their parent atoms.

\section{Comment}

Schiff base compounds and their complexes have been the centre of attraction for many researcher in recent years [7, 8]. The Schiff base can be used as a more or less flexible ligand $[9,10]$. It is usually coordinated by the $O$ atom of the substituted phenol group and the $\mathrm{N}$ atom of the azomethine group to the transition metal [11-13]. We have designed and prepared another Schiff base compound, and we predict that it has useful properties and applications, for example for copper(II) complexes [14-16].

The single crystal structure of the title compound was determined by X-ray crystallography. All geometric parameters are in the typical ranges. There is a strong intramolecular $\mathrm{O} 1-\mathrm{H} 1 \cdots \mathrm{N} 1$ hydrogen bond interaction $(\mathrm{d}(\mathrm{N} 1 \cdots \mathrm{H} 1)=1.823 \AA \mathrm{d}(\mathrm{O} 1-\mathrm{H} 1)=0.836(10) \AA$ and $\mathrm{d}(\mathrm{O} 1 \cdots \mathrm{N} 1)=2.594 \AA$ ) in the title structure (cf. the figure).

\section{References}

1. Bruker. APEX2, SAINT and SADABS. Bruker AXS Inc., Madison, WI, USA (2012).

2. Sheldrick, G. M.: A short history of SHELX. Acta Crystallogr. A64 (2008) 112-122.

3. Dolomanov, O. V.; Bourhis, L. J.; Gildea, R. J.; Howard, J. A. K.; Puschmann, H.: OLEX2: A complete structure solution, refinement and analysis program. J. Appl. Crystallogr. 42 (2009) 339-341.

4. Ma, J. X.; Wei, P.; Li, Q. L.; Fu, T.; Zhao, J. X.; Zhao, L.: Crystal structure of $(E)-1-(4-((E)$-2-bromo-6-hydroxybenzylidene) amino)phenyl)ethan-1-one $O$-methyl oxime, $\mathrm{C}_{16} \mathrm{H}_{15} \mathrm{BrN}_{2} \mathrm{O}_{2}$. Z. Kristallogr. NCS 235 (2020) 19-20.

5. Zhao, J. X.; Zhao, L.; Li, P. P.; Wang, F.; An, Q. Q.: Crystal structure of (E)-1-(4-(( $(E)$-5-bromo-2-hydroxybenzylidene) amino)phenyl)ethan-1-one $O$-methyl oxime, $\mathrm{C}_{16} \mathrm{H}_{15} \mathrm{BrN}_{2} \mathrm{O}_{2}$. Z. Kristallogr. NCS 232 (2017) 731-732.

6. Fu, T.; Ma, J. X.; Li, Q. L.; Wei, P.; Zhao, J. X.; Zhao, L.: Crystal structure of $(E)$-1-\{4-[(4-fluoro-2-hydroxybenzylidene)amino] phenyl\}ethanone $O$-methyl oxime, $\mathrm{C}_{16} \mathrm{H}_{15} \mathrm{FN}_{2} \mathrm{O}_{2}$. Z. Kristallogr. NCS 235 (2020) 293-295.

7. Ma, J. X.; Li, Q. L.; Li, P. P.; Zhao, J. X.; Zhao, L.: Synthesis and crystal structure of $(E)-1-(4-((E)-5$-chloro-2hydroxybenzylidene)amino)phenyl)ethan-1-one $O$ methyloxime, $\mathrm{C}_{16} \mathrm{H}_{15} \mathrm{ClN}_{2} \mathrm{O}_{2}$. Z. Kristallogr. NCS 233 (2018) 463-464.

8. Zhao, L.; Wang, F.; An, Q. Q.; Zhao, J. X.: Crystal structure of (E)-1-(4-(((E)-3,5-dichloro-2-hydroxybenzylidene)amino)phenyl) ethan-1-one $O$-ethyl oxime, $\mathrm{C}_{17} \mathrm{H}_{16} \mathrm{Cl}_{2} \mathrm{~N}_{2} \mathrm{O}_{2}$. Z. Kristallogr. NCS 231 (2016) 1045-1046.

9. Zhao, L.; Zhao, J. X.; An, Q. Q.; Wang, F.: Crystal structure of (E)-1-(4-(( $E)$-3,5-dibromo-2-hydroxybenzylidene)amino)phenyl) ethan-1-one $O$-methyl oxime, $\mathrm{C}_{17} \mathrm{H}_{16} \mathrm{Br}_{2} \mathrm{~N}_{2} \mathrm{O}_{2}$. Z. Kristallogr. NCS 231 (2016) 1053-1054.

10. Fu, T.; Ma, J. X.; Wei, P.; Li, Q. L.; Zhao, J. X.; Zhao, L.: Synthesis

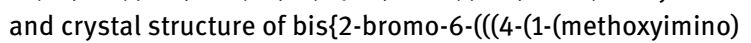
ethyl)phenyl)imino)methyl) phenolato- $\left.\mathrm{K}^{2} \mathrm{~N}, \mathrm{O}\right\}$ cobalt(II)dichloromethane(1/1), $\mathrm{C}_{34} \mathrm{H}_{32} \mathrm{Br}_{2} \mathrm{Cl}_{4} \mathrm{CoN}_{4} \mathrm{O}_{4}$. Z. Kristallogr. NCS 235 (2020) 59-60.

11. Sun, Y. X.; Zhao, Y. Y.; Li, C. Y.; Yu, B.; Guo, J. Q.; Li, J.: Supramolecular cobalt(II) and copper(II) complexes with Schiff base ligand: syntheses, characterizations and crystal structures. Chin. J. Inorg. Chem. 32 (2016) 913-920.

12. Li, Q. L.; Li, P. P.; Ma, J. X.; Zhao, J. X.; Zhao, L.: Crystal structure of bis\{2-((E)-((4-((E)-1-(methoxy imino)ethyl)phenyl)imino) methyl)phenolato- ${ }^{2} \mathrm{~N}, \mathrm{O}$ \}zinc(II), $\mathrm{C}_{32} \mathrm{H}_{30} \mathrm{~N}_{4} \mathrm{O}_{4} \mathrm{Zn}$. Z. Kristallogr. NCS 233 (2018) 637-639.

13. Sun, Y. X.; Lu, R. E.; Li, X. R.; Zhao, Y. Y.; Li, C. Y.: A Schiff base ligand containing oxime group and its $\mathrm{Cu}(\mathrm{II})$ complex: syntheses and supramolecular structures. Chin. J. Inorg. Chem. 31 (2015) 1055-1062.

14. Sun, Y. X.; Zhao, Y. Y.; Li, C. Y.; Yu, B.; Guo, J. Q.; Li, J.: Supramolecular cobalt(II) and copper(II) complexes with Schiff base ligand: syntheses, characterizations and crystal structures. Chin. J. Inorg. Chem. 32 (2016) 913-920.

15. Sun, Y. X.; Lu, R. E.; Li, X. R.; Zhao, Y. Y.; Li, C. Y.: A Schiff base ligand containing oxime group and its $\mathrm{Cu}(\mathrm{II})$ complex: syntheses and supramolecular structures. Chin. J. Inorg. Chem. 31 (2015) 1055-1062.

16. Zhao, L.; An, Q. Q.; Zhao, J. X.; Li, P. P.; Wang, F.: Crystal structure of bis(2-((E)-((4-((E)-1-(ethoxyimino) ethyl)phenyl)imino) methyl)-5-methoxyphenolato- $\mathrm{K}^{2} \mathrm{~N}, \mathrm{O}$ ) copper(II), $\mathrm{C}_{36} \mathrm{H}_{38} \mathrm{CuN}_{4} \mathrm{O}_{6}$. Z. Kristallogr. NCS 232 (2017) 595-597. 